\title{
Identifying adolescents with high fasting glucose: The importance of adding grandparents' data when assessing family history of diabetes
}

\author{
Mariana Brandão ${ }^{\text {a }}$, Carla Lopes ${ }^{\mathrm{a}, \mathrm{b}}$, Elisabete Ramos ${ }^{\mathrm{a}, \mathrm{b}, *}$ \\ a Department of Clinical Epidemiology, Predictive Medicine and Public Health, University of Porto Medical School, Alameda Professor Hernâni Monteiro, 4200 - 319 Porto Portugal \\ b Institute of Public Health, University of Porto (ISPUP), Rua das Taipas, $n^{\circ}$ 135, 4050-600, Porto Portugal
}

\section{A R T I C L E I N F O}

Available online 9 July 2013

Keywords:

Type 2 diabetes mellitus

Adolescent

Abnormal glycemia

Family history

\section{A B S T R A C T}

Objective. To evaluate the role of adding grandparents' data to parental information to the assessment of a family history of diabetes, in order to identify adolescents with high fasting glucose.

Method. In 2003 we evaluated 1276 population-based 13-year-olds, from Porto, Portugal. The history of diabetes in parents and grandparents was collected using self-reported questionnaires and a clinical evaluation was performed, including a fasting blood sample. The 75th percentile of fasting plasma glucose ( $\mathrm{FPG}=91 \mathrm{mg} / \mathrm{dl}$ ) was used to create two groups of participants (high vs. low fasting glucose).

Results. No association was found between family history of diabetes (with or without grandparental data) and a high FPG. The sensitivity to identify individuals with high FPG increased from $7.8 \%$ to $47.9 \%$ when grandparental history was combined with parental data. The positive predictive value was slightly increased ( $25.2 \%$ vs. $27.8 \%$ ) but the specificity dropped ( $91.8 \%$ vs. $56.4 \%)$.

Conclusion. Combining parental with grandparental history increased the number of adolescents with a positive family history of diabetes and also increased the sensitivity to identify adolescents with high FPG. So, even if it determines a decrease in specificity, grandparental data is relevant when screening for high fasting glucose in adolescents.

(c) 2013 Elsevier Inc. All rights reserved.

\section{Introduction}

The prevalence of Type 2 diabetes mellitus (T2DM) among children and adolescents is increasing worldwide, mostly due to the epidemic of overweight and obesity in these age groups (Alberti et al., 2004).

Since more young individuals develop T2DM, their propensity to develop diabetes' related complications is bigger. Hence, identifying adolescents at higher risk of developing the disease must be a priority. Namely, because those complications result from a continuous exposure to high plasma glucose values and can occur even in those individuals with impaired fasting glucose (IFG) or impaired glucose tolerance (Reinehr et al., 2006; WHO, 2006).

In order to minimize these potential consequences, the Consensus Panel of the American Diabetes Association (ADA) recommends screening for T2DM at the age of 10 or at the onset of puberty (when puberty occurs at a younger age), if there is a high probability of developing diabetes. Besides being overweight or obese, one of the screening criteria to identify adolescents is a family history of T2DM in a first- or seconddegree relatives (ADA, 2012). Wei et al. (Wei et al., 2010) conducted a large study that supports this recommendation, suggesting that a

\footnotetext{
* Corresponding author at: Department of Clinical Epidemiology, Predictive Medicine and Public Health, University of Porto Medical School, Alameda Professor Hernâni Monteiro, 4200 - 319 Porto Portugal. Fax: +351 225513653.

E-mail addresses: marbran@gmail.com (M. Brandão), carlal@med.up.pt (C. Lopes), eliramos@med.up.pt (E. Ramos).
}

parental or grandparental history of diabetes is associated with an increased risk of T2DM in children. However, ADA recommendations regarding screening are not widely followed in clinical practice (Drobac et al., 2004; Rhodes et al., 2006; van Esch et al., 2013).

Using family history information as a screening tool is appealing in both clinical and community settings, because it is easy and inexpensive to collect. Unfortunately, among younger adolescents there is a huge probability of misclassification regarding family history based only on parental data, since most of their parents are still in their 40s and 50s and a large proportion of subjects younger than 50 years of age are still undiagnosed (DECODE, 2003). Thus, data about grandparents' history of diabetes may help to minimize this misclassification.

Despite the low prevalence of T2DM at early adolescence, having high fasting plasma glucose (FPG) level, even within the normoglycemic range, is a predictor of type 2 diabetes in younger adulthood (Nguyen et al., 2010). Therefore, the goal of our study is to evaluate the role of adding grandparental data to parental information to the assessment of a family history of diabetes, in order to identify adolescents with high fasting plasma glucose.

\section{Methods}

\section{Participants}

The study was developed as part of the Epidemiological Health Investigation of Teenagers in Porto (EPITeen). Eligible participants were adolescents born in 
1990 and enrolled at public and private schools in Porto during the 2003/2004 school year (Ramos and Barros, 2007). We identified 2787 eligible adolescents, and 2160 (1561 public and 509 private school students) agreed to participate. This resulted in a 77.5\% overall participation, with a similar level in both public (77.7\%) and private schools (77.0\%, $\mathrm{p}=0.709)$.

Ethics

The Ethical Committee of the University Hospital of São João, Porto, approved the study. Written informed consent was obtained from both parents and adolescents.

\section{Data collection}

The evaluation included two self-administered questionnaires and a physical examination. The home questionnaire, answered by adolescents and parents, inquired into demographic, social, behavioral and clinical history. Parents reported their usual weight and height (this information being used to calculate body mass index - BMI) and their smoking status. Based on parents' education, adolescents were classified, taking into account the parent with a higher level of education, and this information was used as a proxy for socioeconomic status.

Information about a medical diagnosis of diabetes was asked separately to the mother, father and each of the grandparents. We have classified Parental History ( $\mathrm{PH}$ ) as: a) positive, when at least one of the adolescent's parents had a diagnosis of diabetes; b) negative, when both parents reported absence of diabetes or $\mathrm{c}$ ) non classifiable, when the available information showed no diagnosis of diabetes, but, at least, one of the parents reported to "be unaware" or did not answer. Total Family History (TFH) was computed taking into account the information on parental and grandparental diagnosis of diabetes. We have classified TFH as: a) positive, when at least one of the adolescent's relatives (parents or grandparents) had a diagnosis of diabetes; b) negative, when all relatives reported absence of diabetes; c) non classifiable, when the available information about relatives showed no diagnosis of diabetes, but there was missing or "unaware" information about, at least, one relative.

A physical examination was performed at school by a team of health professionals. Anthropometrics were obtained with the subject in light indoor clothes and no shoes. Weight was measured using a digital scale Tanita ${ }^{\circledR}$ (in kilograms, to the nearest $0.1 \mathrm{~kg}$ ) and height was measured (in centimeters, to the nearest tenth) using a portable stadiometer. BMI was classified according to the age-specific percentiles developed by the United States Centers for Disease Control and Prevention (Kuczmarski et al., 2002) as overweight (BMI between the 85 th -95 th percentile) and obese (BMI $\geq 95$ th percentile).

A 12-h overnight fasting blood sample was drawn from consenting participants. Plasma glucose was measured using automatic standard routine enzymatic methods. Insulin was measured by radioimmunoassay (Coat-A-CountR, Diagnostic Products Corporation, Los Angeles, California, USA). Insulin resistance was assessed by the Homeostatic Model Assessment (HOMA-IR) method, based on fasting glucose and insulin concentrations (Matthews et al., 1985) Insulin sensitivity was determined by the Quantitative Insulin Sensitivity Check Index method (QUICKI)(Katz et al., 2000).

We classified adolescents according to their FPG levels, and adolescents equal or above the 75th percentile of FPG (which is $91 \mathrm{mg} / \mathrm{dl}$ ) were classified as "high FPG".

\section{Statistical analyses}

Proportions were compared using the Chi-square test or Fisher's exact test. Means were presented as mean (standard error) and compared using the T-Student or One-Way ANOVA; additionally, to compare values according to the 3 categories of family history, the Bonferroni method was used for post-hoc comparisons. Odds Ratio and 95\% Confidence Intervals were estimated by unconditional Logistic Regression and used to estimate the magnitude of the associations between high FPG and adolescent characteristics.

To estimate the ability to identify high FPG in adolescents based on their family history of diabetes, we aggregated the "Non-classifiable" family histories with the "Negative" family histories. In a clinical setting, the physician has to classify the family history of diabetes as "positive" or "negative", and the nonclassifiable family histories are usually counted as negative.

Statistical significance was accepted at a p value of 0.05 . All analyses were performed using SPSS version 18.0 (SPSS Inc, Chicago, Illinois).

\section{Results}

From the 2160 participants, 2054 participants performed the physical examination, but 388 did not agree to a venopuncture, 274 were not fasting at the time of examination and 6 samples were lost during the handling procedures. Of the 1386 participants with blood sample, 105 did not hand back their parents questionnaire. Among the 1281 adolescents with complete information, five participants had a previous diagnosis of diabetes. So, we analyzed data from 1276 participants. Comparing with participants who were not considered in the analyses, those with complete information presented a higher proportion of girls and adolescents whose parents had a higher economic status (measured by their higher education and their enrollment to a private school). No significant differences were found between those groups in terms of family history of diabetes in parents or grandparents, categories of BMI or age at menarche (data not shown).

From the 1276 participants, 332 had fasting plasma glucose above the 75 th percentile $(91 \mathrm{mg} / \mathrm{dl})$. Those with higher FPG presented higher fasting insulin values, were significantly more insulin resistant (HOMA-IR), and had lower insulin sensitivity (QUICKI) (Table 1).

About family history of diabetes, $5(0.4 \%)$ adolescents had both parents with a diagnosis of diabetes, while in $53(4.2 \%)$ only the mother had diabetes. About grandparental history, 368 adolescents (28.8\%) had just one grandparent with diabetes, 130 (10.2\%) had two and only 4 $(0.3 \%)$ had the four grandparents diagnosed with diabetes.

No significant differences were found on mean fasting plasma glucose according to Parental Family History (data not shown). Regarding Total Family History, mean (standard deviation) of fasting plasma glucose according to the family history status was $86.4 \mathrm{mg} / \mathrm{dL}$ (7.2) for Negative, $85.5 \mathrm{mg} / \mathrm{dL}$ (9.0) for Positive and $83.5 \mathrm{mg} / \mathrm{dL}$ (11.3) for Non Classifiable. Using the Bonferroni post-hoc test, the difference was significant only for: "Negative" vs. "Non classifiable", p $<0.001$ and "Positive" vs. "Non classifiable", $\mathrm{p}=0.002$. No significant differences were found between those classified as "Negative" versus those classified as "Positive" ( $p=0.494$ ). No significant association was found between the family history of diabetes, considering only parents information or the combination of parental and grandparental data, and high FPG (Table 2).

When we used data only from Parental History, we identified 103 adolescents with a positive family history of diabetes ( $8 \%$ of participants). However, combining both parental and grandparental history (Total Family History), 468 (37\% of the total participants) adolescents were additionally identified as having a positive history of diabetes (406 that were initially classified as negative and 62 that were "non classifiable" based on parents' data). Nevertheless, 276 (28\%) of adolescents who were classified as having a "negative family history" were re-classified as "non classifiable" when we considered grandparents' data (Fig. 1).

We calculated the sensitivity, specificity and positive predictive value (PPV) of the positive family history of diabetes to identify adolescents with high fasting glucose (Table 3). Considering all adolescents, when combining grandparental history with parental History's, the

Table 1

Comparison of FPG, FPI, HOMA-IR and QUICKI according to the categories: Low Fasting Glucose (FPG $<75$ th percentile) and High Fasting Glucose (FPG $\geq 75$ th percentile).

\begin{tabular}{lllll}
\hline & $\begin{array}{l}\text { Total } \\
(\mathrm{n}=1276)\end{array}$ & $\begin{array}{l}\text { Low fasting glucose } \\
(\mathrm{n}=944) \\
\text { Mean }(\mathrm{SE})\end{array}$ & $\begin{array}{l}\text { High fasting glucose } \\
(\mathrm{n}=332)\end{array}$ & p-value $^{\mathrm{a}}$ \\
& Mean $(\mathrm{SE})$ & $9.60(6.27)$ & $<0.001$ \\
\hline${\text { FPI }(\mu \mathrm{IU} / \mathrm{ml})^{\mathrm{b}}}^{\mathrm{b}}$ & $8.22(5.53)$ & $7.73(5.16)$ & $2.28(1.53)$ & $<0.001$ \\
HOMA-IR $^{\mathrm{b}}$ & $1.75(1.23)$ & $1.56(1.05)$ & $0.36(0.08)$ & $<0.001$ \\
QUICKI $^{\text {b }}$ & $0.38(0.09)$ & $0.39(0.09)$ & 0.36 \\
\hline
\end{tabular}

a p-value refers to the comparison between the group with FPG $<75$ th percentile and those with FPG $\geq 75$ th percentile. FPG 75 th percentile $=91 \mathrm{mg} / \mathrm{dl}$. ${ }^{\mathrm{b}}$ FPG: Fasting Plasma Glucose. FPI: Fasting Plasma Insulin; HOMA-IR: HOMA-insulin resistance; QUICKI: quantitative insulin sensitivity check index. 
Table 2

Association of individual and familial adolescents' characteristics with a High Fasting Plasma Glucose (FPG $\geq 75$ th percentile).

\begin{tabular}{|c|c|c|c|c|}
\hline & $\begin{array}{l}\text { Low fasting glucose } \mathrm{e}^{\mathrm{a}} \\
\mathrm{n}(\%)\end{array}$ & $\begin{array}{l}\text { High fasting glucose }{ }^{a} \\
n(\%)\end{array}$ & $\begin{array}{l}\text { Crude OR } \\
(95 \% \mathrm{CI})\end{array}$ & $\begin{array}{l}\text { Adjusted } \mathrm{OR}^{\mathrm{b}} \\
(95 \% \mathrm{CI})\end{array}$ \\
\hline \multicolumn{5}{|l|}{ Sex } \\
\hline Female & $527(55.8)$ & $157(47.3)$ & $0.70(0.54-0.90)$ & $0.71(0.55-0.91)$ \\
\hline \multicolumn{5}{|l|}{ Type of school } \\
\hline Private & $245(26.0)$ & $80(24.1)$ & $0.91(0.68-1.21)$ & $1.01(0.74-1.38)$ \\
\hline \multicolumn{5}{|l|}{ Parents education } \\
\hline$\leq 6$ & $242(25.9)$ & $78(24.0)$ & 1 & 1 \\
\hline $7-9$ & $188(20.2)$ & $90(27.7)$ & $1.11(0.77-1.59)$ & $1.46(1.02-2.08)$ \\
\hline $10-12$ & 249 (26.7) & $83(25.5)$ & $1.64(1.15-2.38)$ & $0.99(0.69-1.41)$ \\
\hline$\geq 12$ & $254(27.2)$ & $74(22.8)$ & $1.14(0.80-1.64)$ & $0.87(0.60-1.25)$ \\
\hline Missing & 11 & 7 & & \\
\hline \multicolumn{5}{|l|}{ Smoking parents } \\
\hline None & $204(22.0)$ & $63(19.4)$ & 1 & 1 \\
\hline One & $383(41.3)$ & $144(44.3)$ & $0.89(0.63-1.27)$ & $1.25(0.89-1.77)$ \\
\hline Both & $341(36.7)$ & $118(36.3)$ & $1.09(0.82-1.44)$ & $1.15(0.81-1.65)$ \\
\hline Missing & 16 & 7 & & \\
\hline \multicolumn{5}{|l|}{ BMI-Parents } \\
\hline$<24.9$ & $290(32.2)$ & $98(31.1)$ & 1 & 1 \\
\hline $25-29.9$ & $437(48.4)$ & $151(47.9)$ & $1.02(0.76-1.37)$ & $0.90(0.62-1.30)$ \\
\hline$\geq 30$ & $175(19.4)$ & $66(21.0)$ & $1.12(0.78-1.61)$ & $0.89(0.63-1.25)$ \\
\hline Missing & 42 & 17 & & \\
\hline \multicolumn{5}{|c|}{ Regular practice of sports } \\
\hline No & $468(50.7)$ & $158(48.3)$ & 1 & 1 \\
\hline Yes & $455(49.3)$ & $169(51.7)$ & $0.91(0.71-1.17)$ & $1.13(0.86-1.48)$ \\
\hline Missing & 21 & 5 & & \\
\hline \multicolumn{5}{|l|}{ Age at menarche ${ }^{c}$} \\
\hline $8-11$ & $176(39.4)$ & $34(28.1)$ & 1 & 1 \\
\hline 12 & $174(38.9)$ & $60(49.6)$ & $0.54(0.33-0.86)$ & $0.52(0.32-0.84)$ \\
\hline$\geq 13$ or not yet & $158(31.1)$ & $57(37.8)$ & $0.96(0.63-1.46)$ & $0.94(0.61-1.43)$ \\
\hline Missing & 19 & 6 & & \\
\hline \multicolumn{5}{|l|}{ Categories of BMI } \\
\hline$<85$ th percentile & $691(73.4)$ & $232(69.9)$ & 1 & 1 \\
\hline 85th-95th perc. & $158(16.8)$ & $59(17.8)$ & $1.11(0.80-1.55)$ & $1.11(0.79-1.56)$ \\
\hline$>95$ th percentile & $93(9.9)$ & $41(12.3)$ & $1.31(0.88-1.95)$ & $1.30(0.87-1.94)$ \\
\hline Missing & 2 & 0 & & \\
\hline \multicolumn{5}{|l|}{ Parental History } \\
\hline Negative & 707 (74.9) & $257(77.4)$ & 1 & 1 \\
\hline Positive & $77(8.2)$ & $26(7.8)$ & $0.93(0.58-1.48)$ & $0.91(0.57-1.47)$ \\
\hline Non classifiable & $160(16.9)$ & $49(14.8)$ & $0.84(0.59-1.20)$ & $0.76(0.53-1.11)$ \\
\hline \multicolumn{5}{|l|}{ Total family history } \\
\hline Negative & $214(22.7)$ & $68(20.5)$ & 1 & 1 \\
\hline Positive & $412(43.6)$ & $159(47.9)$ & $0.96(0.68-1.37)$ & $1.17(0.83-1.65)$ \\
\hline Nonclassifiable & $318(33.7)$ & $105(31.6)$ & $1.17(0.88-1.56)$ & $0.95(0.65-1.37)$ \\
\hline
\end{tabular}

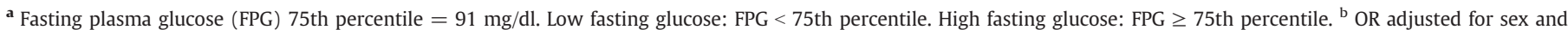
parents education. ${ }^{\mathrm{c}}$ Age at menarche refers only to girls and has a total $\mathrm{n}=684$.

sensitivity increased from $7.8 \%$ to $47.9 \%$. On the other hand, specificity decreased from $91.8 \%$ to $56.4 \%$. Similar results were found considering only obese or overweight adolescents. The PPV, this is, the probability of having high fasting glucose given they have a positive history, is similar between Parental History and Total Family History.

\section{Discussion}

There is an important debate around which should be the cut-point for the definition of impaired fasting glucose (IFG). As the prevalence of IFG and diabetes in our sample was low (4.1\%, using ADA criteria (ADA, 2012), we decided to use the FPG's 75th percentile of our sample to identify those in higher risk to develop IFG or diabetes and its complications. This option is in conformity with data showing that an elevated FPG level in childhood, even within the normoglycemic range, is a predictor of type 2 diabetes in younger adulthood (Nguyen et al., 2010). This option was also supported by the results showing that those with a FPG $\geq 75$ th percentile had a significant worse insulin profile regarding data from insulin resistance and sensitivity indexes.

Surprisingly, there was no relationship between adolescents' categories of BMI and high FPG. However, we found that overweight and obese adolescents had higher fasting plasma insulin levels and were more insulin resistant (data not shown). This is consistent with the fact that obesity is considered the major cause of peripheral insulin resistance in childhood and it is strongly related to the development of altered glucose metabolism (Cali and Caprio, 2008). Probably, the absence of association was due to the lack of power, since we have less than $10 \%$ of obese adolescents.

\section{Family History and High Fasting Plasma Glucose}

No association was found between a positive Family History of diabetes and a high fasting glucose. This is in disagreement with Wei et al (Wei et al., 2010), that showed a higher risk for T2DM in adolescents with a family history of diabetes. However, we need to reinforce that our sample was healthy and population-based and that we used high fasting glucose as outcome, instead of T2DM.

Other possible explanation could be related with the validity of the information on parents' and grandparents' data, since most of the home questionnaires were answered by the mother. However, data from the Framingham Offspring Study (Murabito et al., 2004) showed that both positive and negative parental history reports of diabetes were reliable. Also, Saito et al (Saito et al., 2009), showed that a questionnaire was a reliable method of collecting students' family history, including data on grandparents. 


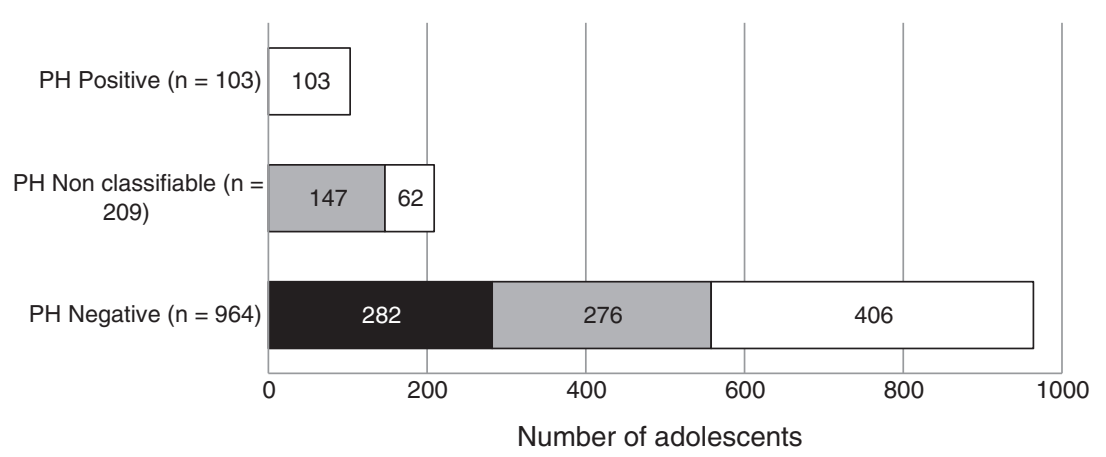

Maintained as Negative $\quad \square$ Changed or maintained as Non classifiable $\quad \square$ Changed or maintained as Positive

Fig. 1. Changing in the classification of Family History of Diabetes by adding grandparental data to Parental History (PH) alone.

\section{Parental History vs. Total Family History}

We found that combining parental with grandparental history of diabetes lead to a 5.5 fold increase in the identification of adolescents with a positive family history. This could be explained by the fact that these adolescents' parents are still young (fathers' mean age: 44.8 years and mothers' mean age: 42.0 years), so most of those that will have a diagnosis of diabetes, do not have it yet. On the other hand, the use of grandparental data had the limitation of increasing the amount of adolescents classified as "Non classifiable" due to the missing information. In this case, they were classified according to parental history, so that does not imply lost of information when using total family history, comparing with using only parental data.

There was a huge increase in the sensitivity to identify adolescents with high fasting glucose when we used Total Family History instead of just Parental History, both in all subjects and in obese/overweight adolescents. However, specificity dropped. Nevertheless, as the aim is to use family history as a screening tool, it is preferable to give way to a higher sensitivity, since adolescents can be submitted to blood tests and a tightened follow-up. Additionally, even a false positive may benefit, as primary interventions have the potential to be protective for a large set of other diseases (Williams et al., 2001).

Adding grandparental information did not decrease PPV - actually increased it a little, but not significantly. So, combining parental with parental information may even improve family history's PPV.

Previous studies found that the risk to develop T2DM varied by the number and type of family members with diabetes (Hemminki et al., 2010; Meigs et al., 2000; Wei et al., 2010). We would like to test this issue in our sample, nonetheless among those with high FPG, we only had 159 adolescents with a positive Total Family History, so there are not enough cases to perform a strong analysis by number and type of affected family members.

We decided to exclude those adolescents with a previous diagnosis of diabetes to test the characteristics of family history to identify adolescents at risk, because the diagnosis of a disease can change the knowledge on family history, which could falsely improve the performance of family history as a screening tool.

\section{Study limitations}

Glucose assays were only run once, not in duplicate: a systematic review which assessed the reproducibility of IFG in adults showed that the $\mathrm{k}$ coefficients indicated only a moderate agreement for IFG (0.44 and 0.56) (Balion et al., 2007). An Oral Glucose Tolerance Test (OGTT) could add some information. However, it would be impossible to perform an OGTT on such a large sample and reduce the sample could affect the external validity.

\section{Strengths}

A major strength of our study is its relatively large sample size, which was taken from a nonclinical population. In Portugal, education is mandatory till 15-year-old, so recruiting 13-year-old adolescents from school gave us the better sample basis. Besides, we have a good rate of participation and there were almost no differences between those participants not considered in the analyses and those with complete information, which minimizes a possible selection bias. Therefore, we have a high confidence that our results give a good perspective of our teenage population.

Family history's assessment was made before the clinical evaluation (including fasting blood sample): the adolescent and his relatives were not aware of his glycemic status when the questionnaire was answered, so there is no awareness bias.

\section{Conclusion}

We could not find an association between a family history of diabetes (only parental or combining parental plus grandparental) and a high fasting glucose. However, combining parental with grandparental history (Total Family History) leads to a 5.5 fold increase in the identification of adolescents with a positive family history. Also, the sensitivity to identify adolescents with high fasting glucose increased from $7.8 \%$ to $47.9 \%$ using Total Family History. Although its specificity is much lower than Parental History's, we know that in a clinical setting, family history is used as a screening tool. So, we can assume that

Table 3

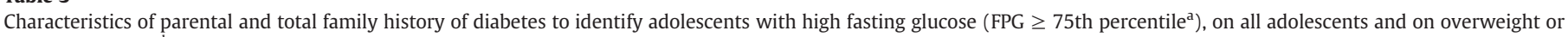
obese adolescents $^{\mathrm{b}}$ - percentage estimates and $(95 \% \mathrm{CI})$.

\begin{tabular}{|c|c|c|c|c|}
\hline & & Sensitivity & Specificity & $\mathrm{PPV}^{\mathrm{c}}$ \\
\hline \multirow{2}{*}{ All adolescents } & Parental history & $7.8 \%(5.2-11.3)$ & $91.8 \%(89.9-93.5)$ & $25.2 \%(17.2-34.7)$ \\
\hline & Total family history & $47.9 \%(42.4-53.4)$ & $56.4 \%(53.1-59.5)$ & $27.8 \%(24.2-31.7)$ \\
\hline \multirow[t]{2}{*}{ Only obese or overweight } & Parental history & $10.0 \%(4.9-17.6)$ & $90.5 \%(86.2-93.8)$ & $29.4 \%(27.2-31.6)$ \\
\hline & Total family history & $45.0 \%(35.0-55.3)$ & $51.4 \%(45.0-57.7)$ & $26.8 \%(20.2-34.2)$ \\
\hline
\end{tabular}

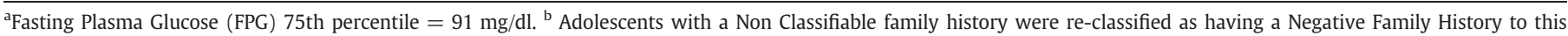
analysis. ${ }^{\mathrm{C}}$ PPV: Positive Predictive Value. 
the use of grandparental information adds relevant information on adolescent screening for high fasting glucose.

\section{Conflict of interest statement}

The authors declare that there are no conflicts of interest.

\section{Acknowledgments}

We gratefully acknowledge grants from Fundação para a Ciência e Tecnologia (the Portuguese National institute for the scientific research) for financial support for our research (FCOMP-01-0124-FEDER-015750). This funding source had no role in any part of the study.

The authors are also grateful to the anonymous reviewers of this article, for insightful comments on the original version of the manuscript.

\section{References}

ADA, 2012. Standards of medical care in diabetes-2012. Diabetes Care 35 (Suppl. 1), S11-S63.

Alberti, G., Zimmet, P., Shaw, J., Bloomgarden, Z., Kaufman, F., Silink, M., 2004. Type 2 diabetes in the young: the evolving epidemic: the international diabetes federation consensus workshop. Diabetes Care 27, 1798-1811.

Balion, C.M., Raina, P.S., Gerstein, H.C., et al., 2007. Reproducibility of impaired glucose tolerance (IGT) and impaired fasting glucose (IFG) classification: a systematic review. Clin. Chem. Lab. Med. 45, 1180-1185.

Cali, A.M., Caprio, S., 2008. Prediabetes and type 2 diabetes in youth: an emerging epidemic disease? Curr. Opin. Endocrinol. Diabetes Obes. 15, 123-127.

DECODE, 2003. Age- and sex-specific prevalences of diabetes and impaired glucose regulation in 13 European cohorts. Diabetes Care 26, 61-69.

Drobac, S., Brickman, W., Smith, T., Binns, H.J., 2004. Evaluation of a type 2 diabetes screening protocol in an urban pediatric clinic. Pediatrics 114, 141-148.

Hemminki, K., Li, X., Sundquist, K., Sundquist, J., 2010. Familial risks for type 2 diabetes in Sweden. Diabetes Care 33, 293-297.
Katz, A., Nambi, S.S., Mather, K., et al., 2000. Quantitative insulin sensitivity check index: a simple, accurate method for assessing insulin sensitivity in humans. J. Clin. Endocrinol. Metab. 85, 2402-2410.

Kuczmarski, R.J., Ogden, C.L., Guo, S.S., et al., 2002. 2000 CDC Growth Charts for the United States: methods and development. Vital Health Stat 11 1-190.

Matthews, D.R., Hosker, J.P., Rudenski, A.S., Naylor, B.A., Treacher, D.F., Turner, R.C., 1985 Homeostasis model assessment: insulin resistance and beta-cell function from fasting plasma glucose and insulin concentrations in man. Diabetologia 28, 412-419.

Meigs, J.B., Cupples, L.A., Wilson, P.W., 2000. Parental transmission of type 2 diabetes: the Framingham Offspring Study. Diabetes 49, 2201-2207.

Murabito, J.M., Nam, B.H., D'Agostino Sr., R.B., Lloyd-Jones, D.M., O'Donnell, C.J., Wilson, P.W., 2004. Accuracy of offspring reports of parental cardiovascular disease history: the Framingham Offspring Study. Ann. Intern. Med. 140, 434-440.

Nguyen, Q.M., Srinivasan, S.R., Xu, J.H., Chen, W., Berenson, G.S., 2010. Fasting plasma glucose levels within the normoglycemic range in childhood as a predictor of prediabetes and type 2 diabetes in adulthood: the Bogalusa Heart Study. Arch. Pediatr. Adolesc. Med. 164, 124-128.

Ramos, E., Barros, H., 2007. Family and school determinants of overweight in 13-yearold Portuguese adolescents. Acta Paediatr. 96, 281-286.

Reinehr, T., Kiess, W., de Sousa, G., Stoffel-Wagner, B., Wunsch, R., 2006. Intima media thickness in childhood obesity: relations to inflammatory marker, glucose metabolism, and blood pressure. Metabolism 55, 113-118.

Rhodes, E.T., Finkelstein, J.A., Marshall, R., Allen, C., Gillman, M.W., Ludwig, D.S., 2006 Screening for type 2 diabetes mellitus in children and adolescents: attitudes, barriers, and practices among pediatric clinicians. Ambul. Pediatr. 6, 110-114.

Saito, T., Nanri, S., Saito, I., 2009. Reliability of family history of lifestyle-related diseases on questionnaire. Pediatr. Int. 51, 514-519.

van Esch, S.C., Heideman, W.H., Cleijne, W., Cornel, M.C., Snoek, F.J., 2013. Health care providers' perspective on using family history in the prevention of type 2 diabetes: a qualitative study including different disciplines. BMC Fam. Pract. 14, 31.

Wei, J.N., Li, H.Y., Wang, Y.C., et al., 2010. Detailed family history of diabetes identified children at risk of type 2 diabetes: a population-based case-control study. Pediatr. Diabetes 11, 258-264.

WHO, 2006. Definition and diagnosis of diabetes mellitus and intermediate hyperglycemia: report of a WHO/IDF consultation. World Health Organization, Geneva.

Williams, R.R. Hunt, S.C. Heiss, G, et al. 2001. Usefulness of cardiovascular family history data for population-based preventive medicine and medical research (the Health Family Tree Study and the NHLBI Family Heart Study). Am. J. Cardiol. 87 129-135. 